\title{
Evolução do baixo peso ao nascer no estado de Alagoas entre 1998 a 2018
}

\author{
Evolution of low weight at born in the state of Alagoas between 1998 to 2018 \\ Evolución de los nacidos de bajo peso en el estado de Alagoas entre 1998 al 2018
}

Recebido: 02/02/2022 | Revisado: 06/02/2022 | Aceito: 13/02/2022 | Publicado: 19/02/2022

Lídia Bezerra Barbosa

ORCID: https://orcid.org/0000-0003-1883-0734 Universidade Federal de Alagoas, Brasil

E-mail: bezerrabarbosa@gmail.com

Nancy Borges Rodrigues Vasconcelos

ORCID: https://orcid.org/0000-0002-4315-1314 Universidade Federal de Alagoas, Brasil

E-mail: nancymedvet@ hotmail.com

Larissa Feitosa dos Santos

ORCID: https://orcid.org/0000-0002-7193-4076 Universidade Federal de Alagoas, Brasil E-mail: larissafs96@gmail.com

Ewerton Amorim dos Santos

ORCID: https://orcid.org/0000-0001-8453-017X Universidade de Ciências da Saúde de Alagoas, Brasil E-mail: santos.ew.a@gmail.com

Johnnatas Mikael Lopes

ORCID: https://orcid.org/0000-0002-9679-5287 Universidade Federal do Vale do São Francisco, Brasil E-mail: johnnataslopes2@gmail.com

\begin{abstract}
Resumo
Objetivo: estimar a tendência do peso ao nascer e a sua relação com fatores determinantes no estado de Alagoas, Brasil. Métodos: Trata-se de um estudo ecológico de série temporal cuja amostra foi composta pelos nascidos vivos no Estado de Alagoas no período de 1998 a 2018. A fonte de dados foi o Sistema de Informações sobre nascidos vivos. Os dados foram coletados do Departamento de Informática do Sistema Único de Saúde. Foram coletados dados referentes ao peso ao nascer, características sociodemográficas maternas e relacionadas à gestação. $\mathrm{Na}$ análise de dados utilizou-se o método de regressão polinomial linear e adotou-se um nível de significância de 5\%. Resultados: Foram analisados 1.199.161 nascidos vivos, dos quais 85.375 (7,1\%) estavam com baixo peso ao nascer (BPN). A taxa mais elevada de BPN ocorreu em 2013 (7,6\%). Nascidos vivos de mães com idade $\geq 35$ anos apresentaram uma tendência crescente de BPN com aumento médio anual de $13,4 \%$ dos nascimentos com BPN ( $<<0,001)$. Mães com oito (8) anos ou mais de estudo apresentaram tendência crescente para filhos com BPN no período avaliado ( $<<0,001)$. A idade gestacional entre 22 e 36 semanas apresentou uma tendência crescente do BPN $(p=0,002)$. Conclusão: A evolução do peso ao nascer tende uma estabilização global da prevalência de BPN, entretanto mesmo neste contexto é essencial se avaliar a dimensão do peso ao nascer e os fatores que estão associados a este para fomentar o planejamento de programas e políticas públicas de saúde.
\end{abstract}

Palavras-chave: Peso ao nascer; Sistemas de informação em saúde; Saúde materno-infantil.

\begin{abstract}
Objective: Estimating the trend in birth weight and its relationship with determining factors in the state of Alagoas, Brazil. Methods: This is an ecological time series study whose sample was composed of live births in the State of Alagoas from 1998 to 2018. The source was the Information System on Live Births. Data regarding birth weight, maternal sociodemographic characteristics, and pregnancy-related characteristics were collected from the Department of Informatics of the Unified Health System Data. In the data analysis, the linear polynomial regression method was used and a significance level of 5\% was adopted. Results: 1,199,161 live births were analyzed. 85,375 (7.1\%) were low birth weight (LBW). The highest rate of BPN occurred in 2013 (7.6\%). Live births of mothers aged $\geq 35$ years showed an increasing and significant trend of BPN with an annual mean increase of $13.4 \%$ of births with LBW $(\mathrm{p}<0.001)$. Mothers who had been studied for $(8)$ years or more showed an increasing trend for children with LBW in the evaluated period $(\mathrm{p}<0.001)$. Gestational age between 22 and 36 weeks showed an increasing and significant trend of LBW ( $\mathrm{p}=0.002$ ). Conclusion: It was observed that the evolution of birth weight tends towards an overall stabilization of the prevalence of LBW. However, even in this context, it is essential to assess the dimension of birth weight and the factors associated with it to promote the program planning and public health policies.
\end{abstract}

Keywords: Birth weight; Health information systems; Maternal and child health. 


\begin{abstract}
Resumen
Objetivo: estimar la tendencia del peso al nacer y su relación con los factores determinantes en el estado de Alagoas, Brasil. Métodos: Se trata de un estudio de serie temporal ecológica cuya muestra estuvo compuesta por nacidos vivos en el Estado de Alagoas de 1998 a 2018. La fuente de datos fue el Sistema de Información sobre nacidos vivos. Los datos fueron recolectados del Departamento de Tecnología de la Información del Sistema Único de Salud. Se recogieron datos sobre el peso al nacer, características sociodemográficas maternas y relacionadas con el embarazo. En el análisis de los datos se utilizó el método de regresión polinomial lineal y se adoptó un nivel de significación del 5\%. Resultados: Se analizaron un total de 1.199.161 nacidos vivos, de los cuales $85.375(7,1 \%)$ tuvieron bajo peso al nacer (BPN). La tasa más alta de BPN ocurrió en 2013 (7,6\%). Los nacidos vivos de madres $\geq 35$ años mostraron una tendencia creciente para el BPN con un aumento medio anual del 13,4\% de los nacimientos con BPN (p<0,001). Las madres con ocho (8) años de escolaridad o más mostraron una tendencia creciente a tener hijos con BPN en el período evaluado ( $\mathrm{p}<0,001)$. La edad gestacional entre 22 y 36 semanas mostró una tendencia creciente en el BPN ( $\mathrm{p}=0,002)$. Conclusión: La evolución del peso al nacer tiende a una estabilización global de la prevalencia del BPN, sin embargo, aún en este contexto, es fundamental evaluar la dimensión del peso al nacer y los factores que se asocian a él para promover la planificación de programas y políticas de salud pública.
\end{abstract}

Palabras clave: Peso al nacer; Interoperabilidad de la información en salud; Salud materno-infantil.

\title{
1. Introdução
}

O parâmetro peso ao nascer é utilizado para identificar a proporção da população que se enquadra no grupo classificado como de baixo peso ao nascer (BPN), o qual é um indicador preditor da sobrevivência infantil, visto que as evidências apontam que quanto menor o peso ao nascer, maior as chances de morte precoce (Pinto, 2010). O BPN é definido pela Organização Mundial de Saúde (World Health Organization - WHO, 1995) como peso ao nascimento inferior à 2500 gramas.

Dados apontam que a estimativa de BPN no mundo fique em torno de mais de 20 milhões de nascimentos por ano correspondendo a um percentual de 15 a 20\% dos nascimentos (WHO- World Health Organization, 2014). Um estudo realizado com dados de diferentes países apontou uma prevalência global de BPN em 2015 de 14,6\% (IC 95\%:12,4-17,1), representando uma redução na prevalência em comparação ao ano 2000 em que a taxa foi de 17,5\% (IC 95\%: 14,1-21,3) (Blencowe et al., 2019). No Brasil, estudo com dados referentes ao período entre 1996 e 2011 apontou uma taxa de 8,0\% de nascidos vivos com BPN nas 26 capitais dos estados e no Distrito Federal, sendo a proporção encontrada para a região Nordeste de 7,6\% (de Souza Buriol et al., 2016).

Neste contexto, conhecer a dimensão do peso ao nascer e os fatores que estão associados a este são essenciais para o planejamento de programas e políticas públicas de saúde e nutrição (Franceschini et al., 2003; Kramer, 1987; Viana et al., 2013) como por exemplo os Programas nacionais de suplementação de ferro e vitamina A, a Política nacional de Alimentação e Nutrição, o Programa Bolsa Família, entre outros.

Os fatores que e influenciam o BPN são o tabagismo materno, consumo de drogas na gestação, ausência de assistência pré-natal, baixa estatura materna, doença hipertensiva específica gestacional, idade materna avançada (acima de 35 anos) ou mães muito jovens (com menos de 20 anos), abortos espontâneos recorrentes (dois ou mais abortos) e história prévia de filho com BPN e/ou PIG - pequeno para idade gestacional (Cristovão \& Pedraza, 2015; Franciotti et al., 2010; Nunes, 2007; Zambonato et al., 2004, 2004).

Nesta perspectiva o desenvolvimento de pesquisas para avaliar a evolução do peso ao nascer e constatar a ocorrência de casos de BPN no Brasil, bem como estabelecer quais as desigualdades socioespaciais existentes nas diferentes Unidades Federadas do território nacional é de fundamental importância. Assim, o objetivo deste estudo foi descrever a evolução do baixo peso ao nascer e avaliar os fatores determinantes deste no estado de Alagoas, Brasil, no período de 1998 a 2018.

\section{Metodologia}

Trata-se de um estudo do tipo ecológico de série temporal cuja amostra será composta pelo total de nascidos vivos de 
mães residentes no Estado de Alagoas no período de 1998 a 2018, tendo como fonte os dados disponíveis no SINASC do Ministério da Saúde (MS).

O estado de Alagoas está localizado no Nordeste brasileiro, ocupando uma área de $27.767 \mathrm{~km}^{2}$. O estado é formado por 102 municípios, divididos em 10 regiões de saúde, entre as quais na $1^{\mathrm{a}}$ região se encontra a sua capital Maceió, sendo o município mais populoso. A A população total deste Estado de acordo com dados de estimativa do Tribunal de Contas da União para o ano de 2019 foi de 3.337.357 habitantes. O Índice de Desenvolvimento Humano (IDHM) de Alagoas é de 0,683, o que o deixa na faixa de desenvolvimento humano médio - IDHM entre 0,600 e 0,699 (PNUD et al., 2020).

Foram excluídos do estudo os recém-nascidos com os registros de peso ao nascer ignorado e de peso ao nascer < 500 .

Os dados foram coletados do Departamento de Informática do Sistema Único de Saúde (DATASUS. http://datasus.saude.gov.br/) a partir das informações contidas no banco de dados do SINASC, o qual dispõe das variáveis existentes nas Declarações de Nascidos Vivos; e, das estimativas do Instituto Brasileiro de Geografia e Estatística (IBGE).

A variável dependente analisada foi o peso ao nascer, o qual foi classificado de acordo com o preconizado pela Organização Mundial de Saúde (World Health Organization - WHO, 1995): baixo peso< 2500g; peso adequado: 2500-3999g; e peso elevado $\geq 4000 \mathrm{~g}$. Tendo em vista a relevância do BPN como problema de saúde pública, as variáveis independentes foram analisadas em função deste. As variáveis independentes foram compostas por informações sociodemográficas maternas: Idade (10 a 19, 20 a 34, 35 anos ou mais), escolaridade (nenhuma, 1 a 7 anos de estudo, 8 ou mais anos de estudo e informação ignorada), situação conjugal (casada, não casada e informação ignorada); e, informações relacionadas a gestação: número de consultas pré-natal ( 3 ou menos, 4 a 6 consultas, $\geq 7$ consultas e aquelas com informações ignoradas), tipo de parto ( vaginal, cesáreo e ignorado) e idade gestacional (22 a 36 semanas, 37 ou mais semanas e ignorado). Para as regiões de saúde o desfecho foi analisado seguindo a estratificação por região da $1^{\mathrm{a}}$ a $10^{\mathrm{a}}$.

Para o cálculo das proporções de incidência acumulada dos recém-nascidos por classificação do peso ao nascer foi realizada a razão entre o número de nascidos vivos para cada categoria de peso pelo total de nascimentos em cada ano, multiplicado por 100.000 .

No caso das análises para as taxas de prevalência para cada Região de Saúde, os cálculos foram realizados considerando o total de nascidos por categoria de peso ao nascer por ano avaliado, então, utilizou-se a razão entre o número de nascidos vivos para cada categoria de peso, por região de saúde, de acordo com o local de residência materna, pelo total de nascimentos em cada ano por região de saúde, multiplicado por 100.

Para análise da tendência dos dados utilizou-se o modelo de regressão polinomial linear, segundo a equação $y=\beta 1 \mathrm{x}+$ $\beta 0$, sendo o $\beta 1$ interpretado como a velocidade do aumento ou decréscimo do baixo peso ao nascer ao longo do tempo e $\beta 0$ como uma prevalência anual média. O peso ao nascer foi considerado como variável dependente (y) e os anos de estudo como variável independente (x).. Para este modelo foi levado em consideração a análise do gráfico de dispersão. A classificação da tendência se deu da seguinte forma: estacionária a tendência cuja probabilidade foi maior que 0,05 ( $p>0,05)$; crescente a que $\beta 1$ apresentou-se positivo e; decrescente a tendência em que $\beta 1$ teve valor negativo. As análises foram realizadas no Microsoft Excel for Windows $®$. Adotou-se um nível de significância de 5\% para minimizar um erro do tipo I.

Os dados obtidos no presente estudo são provenientes de banco de dados de domínio público, assim tal característica dispensa a apreciação da presente pesquisa por um Comitê de Ética em Pesquisa, de acordo com as Resoluções nº 466/2012 e n 510/2016 do Conselho Nacional de Saúde. 


\section{Resultados}

No período de 1998 a 2018, o estudo avaliou dados de 1.199.161 nascidos vivos de mães residentes no estado de Alagoas que atenderam aos critérios de elegibilidade; dos nascidos vivos avaliados 85.375 (7,1\%) nascidos estavam com BPN e $77.277(6,4 \%)$ com peso elevado.

A Figura 1 apresenta as taxas de peso ao nascer de acordo com a sua classificação segundo o total de nascimentos por ano. Em 1998, a taxa de nascimentos com BPN era de 6,6\%, aumentando para 7,3\% em 2003, com leves aumentos e declínios ao longo do período avaliado, chegando a taxa mais elevada em 2013 de 7,6\%, sendo que a partir deste momento essa taxa vem decrescendo de forma suave.

Figura 1 - Distribuição percentual do baixo peso ao nascer em relação ao total de nascimentos no período de 1998 a 2018 no estado de Alagoas, Brasil.

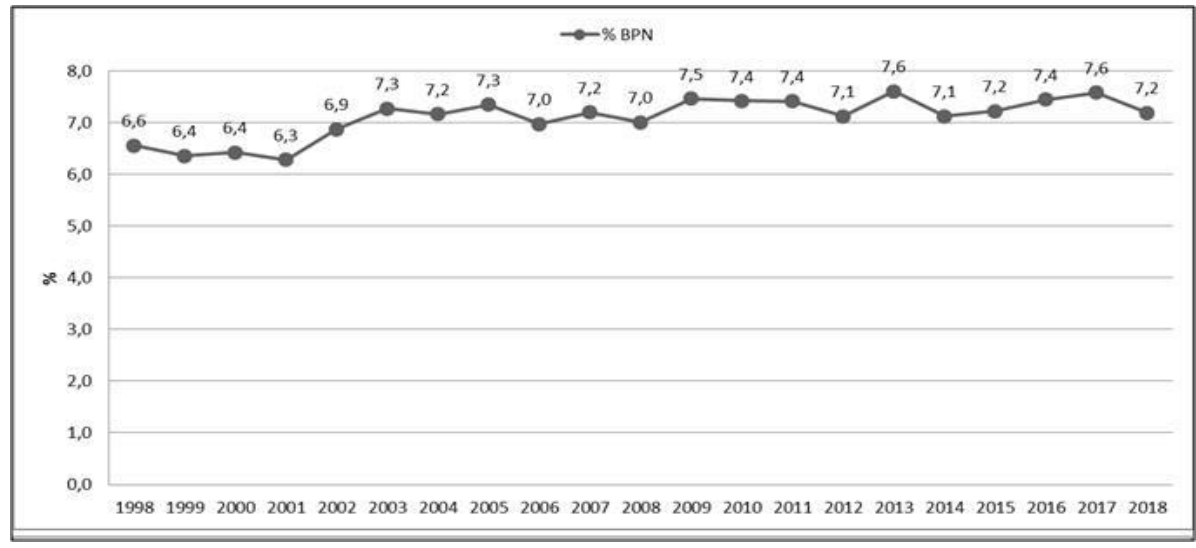

Fonte: Dados da pesquisa. \% BPN- percentual de baixo peso ao nascer.

A Figura 2 apresenta as características de cada uma das 10 regiões de saúde do estado de Alagoas segundo as categorias de peso ao nascer.

Figura 2 - Prevalências de baixo peso ao nascer no estado de Alagoas, Brasil, em relação ao total de nascimentos, segundo a região de saúde no período de 1998 à 2018.

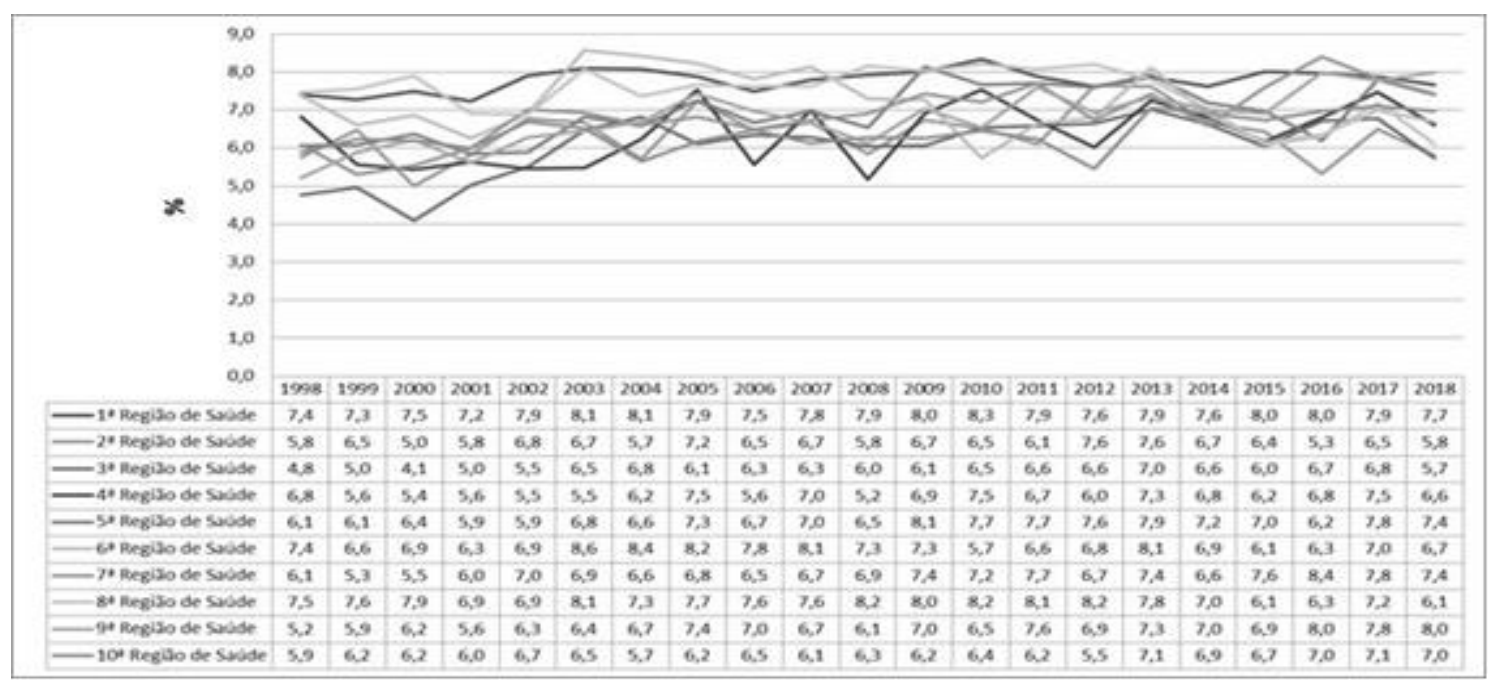

Fonte: Dados da pesquisa. 
As taxas de prevalência variaram em torno de aproximadamente 4,5 a 9\% para cada região de saúde. Em 1998 as prevalências mais altas de BPN eram de 7,4\% para a $1^{\mathrm{a}}$ e $6^{\mathrm{a}}$ regiões de saúde e 7,5\% para a $8^{\mathrm{a}}$ região de saúde; de forma contrária, a $3^{\text {a }}$ região de saúde apresentou a menor taxa de prevalência para este ano $(4,8 \%)$. A maior prevalência de BPN foi em 2003 para a $6^{a}$ região de saúde $(8,6 \%)$. Houve um maior aumento da prevalência para a 9a região de saúde (5,2\% em 1998 para 8,0\% em 2018). Em 2018 a menor prevalência foi da $3^{\text {a }}$ região de saúde (5,7\%), a qual teve sua maior taxa no ano 2013 $(7,0 \%)$ - Figura 2.

A análise de modelagem evidenciou uma tendência marginal negativa com média anual dos nascimentos com peso elevado de -82,17\% ( $p=0,057 ; \mathrm{R}_{\text {ajus= }}^{2}$,27), quando calculado em relação a todos os nascimentos em cada ano - Tabela 1.

Tabela 1 - Modelagem da tendência do peso ao nascer em Alagoas, Brasil, segundo as categorias de peso, entre os anos 1998 e 2018.

\begin{tabular}{lccc}
\hline Variáveis & Modelagem $(\mathbf{a x}+\mathbf{b})$ & $\mathbf{p}$ & Tendência \\
\hline Baixo peso ao nascer & $47,36 \mathrm{x}+6572,3$ & 0,305 & Estacionária \\
Peso adequado & $105,47 \mathrm{x}+84653$ & 0,133 & Estacionária \\
Peso elevado & $-82,17 \mathrm{x}+7241,6$ & 0,057 & Decrescente \\
\hline
\end{tabular}

Fonte: Dados da pesquisa. $\mathrm{p}$ - probabilidade.

A Tabela 2 mostra as equações de tendência para o BPN nas regiões de saúde, em que observando-se individualmente cada região verificou-se uma tendência negativa com significância marginal para a $8^{\mathrm{a}}$ região de saúde $\left(-2,98 \%, \mathrm{R}_{\text {aju }}^{2}=0,57\right.$, p $<0,05)$ indicando uma tendência de declínio do BPN. As outras regiões de saúde apresentaram tendência estacionária ou decrescente, porém sem significância estatística.

Tabela 2 - Modelagem da tendência de baixo peso ao nascer em Alagoas, Brasil, segundo as regiões de saúde, entre os anos 1998 e 2018.

\begin{tabular}{|c|c|c|c|}
\hline Regiões de saúde & Modelagem $(a x+b)$ & $\mathbf{p}$ & Tendência \\
\hline $1^{\text {a }}$ Região de saúde & $28,21 x+2420,8$ & 0,539 & Estacionária \\
\hline $2^{\mathrm{a}}$ Região de saúde & $0,22 x+332,3$ & 0,068 & Estacionária \\
\hline $3^{\mathrm{a}}$ Região de saúde & $2,34 x+425,95$ & 0,190 & Estacionária \\
\hline $4^{\mathrm{a}}$ Região de saúde & $0,24 x+289,6$ & 0,071 & Estacionária \\
\hline $5^{\text {a }}$ Região de saúde & $-1,36 x+497,2$ & 0,083 & Decrescente \\
\hline $6^{\text {a }}$ Região de saúde & $-3,17 x+495,2$ & 0,066 & Decrescente \\
\hline $7^{\text {a }}$ Região de saúde & $16,53 x+928,9$ & 0,155 & Estacionária \\
\hline $8^{\text {a }}$ Região de saúde & $-2,98 x+394,7$ & 0,004 & Decrescente \\
\hline $9^{\text {a }}$ Região de saúde & $4,94 x+484,7$ & 0,301 & Estacionária \\
\hline $10^{a}$ Região de saúde & $2,38 x+302,9$ & 0,746 & Estacionária \\
\hline
\end{tabular}

p - probabilidade. Fonte: dados da pesquisa.

Na Tabela 3, os resultados apresentados estão relacionados ao BPN segundo as características sociodemográficas maternas e relacionadas à gestação. No que se refere a idade materna a modelagem referente a faixa etária $\geq 35$ anos de idade apresentou uma tendência crescente com aumento médio anual de 13,38\% dos nascimentos com BPN $\left(p<0,001 ; R_{a j u}^{2}=0,92\right)$. Para a escolaridade, nascidos vivos de mães com oito (8) anos ou mais de estudo apresentaram uma tendência crescente de BPN, tendo um acréscimo anual de $220,96 \%$ no período avaliado $\left(\mathrm{p}<0,001 ; \mathrm{R}^{2}\right.$ aju $\left.=0,99\right)$; as categorias de escolaridade ignorado e não ter escolaridade apresentaram uma tendência negativa de nascidos vivos com BPN indicando um decréscimo deste entre os anos 1998 a 2018. 
No que se refere ao estado civil, tanto as mulheres casadas quanto as não casadas apresentaram uma tendência crescente de BPN no período analisado. Com relação ao número de consultas pré-natal, observou-se tendência estacionária para o total dos nascimentos, contudo, de maneira controversa, entre os nascimentos em que só foram realizadas três (3) ou menos consultas observou-se uma tendência de decréscimo anual de 59,81\% ( $\left.\mathrm{p}=0,021 ; \mathrm{R}_{\text {aju }}^{2}=0,40\right)$. Para os nascidos vivos de parto cesáreo verificou-se tendência de aumento de BPN com alto poder explicativo do modelo, com acréscimo médio anual de $137,04 \%\left(\mathrm{p}<0,001 ; \mathrm{R}^{2}\right.$ aju $\left.=0,98\right)$. Para a duração da gravidez, a idade gestacional entre 22 e 36 semanas apresentou uma tendência crescente de $114,27 \%$ ao ano de BPN ( $p=0,002 ; \mathrm{R}_{\text {aju }}^{2}=0,62$ ), já nos partos para idade gestacional maior ou igual a 37 semanas verificou-se uma tendência negativa de $-79,35 \%$ ao ano $\left(p=0,048 ; R_{\text {aju }}^{2}=0,29\right)$.

Tabela 3 - Modelagem da tendência de baixo peso ao nascer em Alagoas, Brasil, segundo variáveis sociodemográficas maternas e relacionadas a gestação, entre os anos 1998 e 2018.

\begin{tabular}{|c|c|c|c|c|}
\hline Variáveis & Modelagem $(\mathbf{a x}+\mathbf{b})$ & $\mathbf{p}$ & Tendência & $\mathbf{R}_{\text {ajus }}^{2}$ \\
\hline \multicolumn{5}{|c|}{ Idade materna (anos) } \\
\hline$\geq 10-19$ & $6,18 x+2111,7$ & 0,211 & Estacionária & 0,07 \\
\hline $20-34$ & $30,54 x+3867,1$ & 0,381 & Estacionária & $-0,02$ \\
\hline$\geq 35$ & $13,38 x+551,4$ & $<0,001$ & Crescente & 0,92 \\
\hline \multicolumn{5}{|c|}{ Escolaridade } \\
\hline Nenhuma & $-77,82 x+1513,8$ & 0,009 & Decrescente & 0,49 \\
\hline 1-7anos & $-17,87 x+3481,6$ & 0,085 & Decrescente & 0,21 \\
\hline$\geq 8$ anos & $220,96 x+252,91$ & $<0,001$ & Crescente & 0,99 \\
\hline \multicolumn{5}{|l|}{ Estado civil } \\
\hline Não casada & $365,46 x+2126,4$ & $<0,001$ & Crescente & 0,92 \\
\hline Casada & $158,88 x+1485,9$ & 0,000 & Crescente & 0,71 \\
\hline \multicolumn{5}{|c|}{ Número consultas de pré-natal } \\
\hline$\leq 3$ & $-59,81 x+2552,8$ & 0,021 & Decrescente & 0,40 \\
\hline $4-6$ & $94,94 x+1560,1$ & 0,914 & Estacionária & $-0,11$ \\
\hline$\geq 7$ & $56,29 x+1648,7$ & 0,952 & Estacionária & $-0,11$ \\
\hline \multicolumn{5}{|c|}{ Tipo de parto } \\
\hline Vaginal & $-89,73 x+5183,2$ & 0,008 & Decrescente & 0,50 \\
\hline Cesáreo & $137,04 x+1375,9$ & $<0,001$ & Crescente & 0,98 \\
\hline \multicolumn{5}{|c|}{ Idade gestacional (semanas) } \\
\hline $22-36$ & $114,27 x+1559,6$ & 0,002 & Crescente & 0,62 \\
\hline$\geq 37$ & $-79,35 x+4688,9$ & 0,048 & Decrescente & 0,29 \\
\hline
\end{tabular}

$\mathrm{p}$ - probabilidade; $\mathrm{R}_{\text {ajus- }}^{2}$ Coeficiente de determinação ajustado. Fonte: Dados da pesquisa.

\section{Discussão}

Os resultados deste estudo são capazes de demonstrar por meio de uma série histórica de 21 que o BPN apresentou diminuição da prevalência no estado de Alagoas, sugerindo uma tendência de estabilidade, entretanto a $8^{\mathrm{a}}$ região de saúde do estado de Alagoas demonstrou uma tendência decrescente do BPN ao longo dos anos avaliados.

A $8^{\mathrm{a}}$ região de saúde apresenta o $3^{\circ}$ maior IDH e a $2^{\mathrm{a}}$ menor população do estado de Alagoas; nos anos 2000 e 2010 sofreu redução do Índice de Gini e com isso diminuição das concentrações de rendas (Alagoas. Secretaria de Estado da Saúde, 2014), assim houve melhorias das condições socioeconômicas o que pode ter reflexo sobre essa tendência de decréscimo do BPN. Além disso, a $8^{a}$ região de saúde destacou-se das demais devido ao aumento da proporção de gestantes com 7 ou mais consultas pré-natais (Alagoas. Secretaria de Estado da Saúde de Alagoas, 2017). Sabe-se que o BPN está associado a diversos fatores, um dos principais está ligado às condições socioeconômicas precárias, etnia, idade materna, assistência pré-natal, entre outros fatores associados à saúde materna (Moreira et al., 2018; Pedraza et al., 2014). 
As taxas de BPN vêm, mundialmente, apresentando uma grande variação, sendo que aparecem maiores em países menos desenvolvidos, devido a associação deste fator com as condições socioeconômicas desfavoráveis, apesar da melhora no desenvolvimento socioeconômico gradativo desta população é importante considerar as particularidades regionais na avaliação desses fatores relacionados ao BPN (Guimarães \& Velásquez-Meléndez, 2002).

É importante ressaltar ainda que as proporções de BPN representam a qualidade do atendimento à gestante desde as questões nutricionais até a assistência ao parto, e nesse tocante nas regiões do estado de Alagoas ao longo dos anos 2007 a 2013 houve um aumento das mães que realizaram 7 ou mais consultas pré-natal (Alagoas, 2014). Com relação a este aspecto, um estudo realizado com dados dos Sistemas de Informação em Saúde apontou uma evolução crescente da Atenção Básica em Saúde no Estado de Alagoas, no que se refere à cobertura populacional da Estratégia Saúde da Família (ESF), do Núcleo de Apoio à Saúde da Família (NASF) e do componente da alimentação/nutrição; houve aumento do número de municípios com a ESF implantada entre 1994 a 2013, sendo atingido 100\% dos municípios alagoanos; observou-se um aumento da cobertura da ESF sendo este superior a 380\% no número total da população assistida pela estratégia, em que em 1998 a estimativa de cobertura era de 23\% passando para 74,13\% em 2013 (Da Silva et al., 2014), assim, com esse aumento da assistência na atenção básica houve melhorias na assistência pré-natal em todas as regiões de saúde, contribuindo com a tendência estacionária do BPN.

A tendência crescente para o BPN encontrada para nascidos vivos de mulheres na faixa etária de idade $\geq 35$ anos, e com uma maior escolaridade pode estar relacionada às novas demandas de trabalho que a mulher vem assumindo. Moreira et al. (2018) também verificaram associação semelhante no que se refere a idade materna. Ainda, uma idade mais avançada ( $\geq 35$ anos) está relacionada a um maior risco durante a gravidez, em decorrência da prevalência aumentada de doenças crônicas (especialmente hipertensão arterial, diabetes mellitus e miomas) o que conduz para nascimentos prematuros e BPN (Benzies, 2008; Santos et al., 2009). Com relação à escolaridade, esta é uma condição que pode ter ligação com a idade materna haja vista que tem-se observado que as mulheres têm postergado cada vez mais a gravidez até conseguirem estabilidade profissional, econômica e social, sem perceber que o seu aparelho reprodutor envelhece anatômica e fisiologicamente (T. R. S. R. da Silva, 2003). No Brasil, em estudo realizado entre 1996 a 2011 as regiões Sudeste e Sul possuíam as maiores taxas de BPN em mulheres com 35 anos ou mais; tais regiões ainda se caracterizavam por uma maior escolaridade materna e maior número de consultas de pré-natal, o que foi considerado "paradoxo baixo peso ao nascer" (de Souza Buriol et al., 2016). No que se refere às consultas pré-natal, o resultado aqui obtido é contraditório com o recomendado pelas entidades de saúde haja vista que a observação de que os nascidos vivos de mulheres que realizaram três (3) ou menos consultas apresentaram decréscimo nas proporções de BPN, o que é um paradoxo, pois, a realização de um número maior de consultas de pré-natal é visto como um fator de proteção para saúde materna e do recém de nascido, a medida que por meio destas são repassadas uma maior quantidade de orientações e se tem a oportunidade de realização de procedimentos de qualidade, diminuindo assim o risco da criança nascer com BPN (Barbas et al., 2009). Entretanto, é necessário se avaliar melhor a relação do BPN com o número de consultas de pré-natal, haja vista que tal condição é difícil de ser aferida apenas pela análise do número de consultas, informação esta, somente de cunho quantitativo, que não reflete dados sobre a qualidade do atendimento na consulta, as ações que são desenvolvidas, orientações, entre outras (Chermont et al., 2019; Minaga wa et al., 2006).

Quando o tipo de parto foi analisado, observou-se que nas mulheres com parto cesáreo, houve um aumento de nascidos vivos com BPN. Neste sentido, outros estudos também observaram que o parto cesáreo representou risco para a ocorrência de BPN (Moraes et al., 2012; Moreira et al., 2018). Contudo, vale destacar que é possível que o aumento do BPN relacionado ao tipo de parto cesáreo seja em decorrência do aumento da frequência desse tipo parto. No estado de Alagoas, em 1998 o parto cesáreo representava 20,13\% do total de partos, aumentando para 42,08\% em 2008 e atingindo a frequência de 53,85\% no ano de 2018 (Ministério da Saúde, 2021). 
Os cuidados com parto e indicações clínicas corretas implicam na escolha da via de nascimento. A escolha e o aumento no número de partos cesarianas, não são baseadas em evidências científicas, este fato ocasionou morbidade para o binômio mãe/filho, elevação dos custos com serviços de saúde, o que o tornou-se um problema de saúde pública (Weidle et al., 2014).

O medo da dor no parto, o aprimoramento das técnicas cirúrgicas, rapidez, são argumentos utilizados por alguns obstetras e parturientes, como justificativa da escolha por parto cesariana, como também motivos pessoais (Leguizamon Junior et al., 2013).

Os achados aqui encontrados podem contribuir para atualização do conhecimento sobre o peso ao nascer, e ainda influenciar na tomada de decisão para os gestores responsáveis definirem quais estratégias de saúde pública serão melhor aplicadas de acordo com a realidade em questão.

Este estudo possui algumas limitações, como o uso de dados secundários, os quais estão sujeitos a incompletude e confiabilidade das informações no que tange ao preenchimento dos formulários ou subnotificações; há ainda a impossibilidade de acesso a dados adicionais, como estado nutricional materno pré-gestacional, qualidade do atendimento na consulta prénatal, estado de saúde materna, etc., pois, estas informações não estão disponíveis no banco de dados do SINASC; contudo, salienta-se que poderiam contribuir para o estudo. Entretanto, é importante destacar que os sistemas de informação são fundamentais para o desenvolvimento de pesquisas de maior alcance geográfico.

Por outro lado, o estudo apresenta como ponto forte a utilização da análise de Prais-Winsten, que possibilitou verificar a série temporal das taxas anuais de BPN, o que é importante para a o desenvolvimento de ações de prevenção, recuperação e manutenção da vida em conformidade com a realidade local.

Vale ressaltar que há uma escassez de estudos que avaliaram o peso ao nascer no estado de Alagoas, sendo importante sugerir que estudos populacionais adicionais e mais detalhados sejam realizados com diferentes abordagens, que avaliem o SINASC, incluam uma análise mais crítica da assistência pré-natal e fatores de risco associados às características maternas.

\section{Considerações Finais}

O presente estudo evidenciou que a evolução do peso ao nascer tende à estabilização da prevalência do BPN no estado de Alagoas, e com uma tendência decrescente marginal na $8^{\text {a }}$ região de saúde. Além disso, conclui-se ainda que os nascidos vivos de mulheres com idade mais elevada, de mulheres que possuem uma maior escolaridade e nascidos de parto cesáreo apresentaram uma tendência crescente de BPN. Diante destas observações se faz necessário que haja um aumento das ações e programas de saúde que vêm refletindo positivamente nas condições de saúde dos nascidos vivos e maternas, e ainda que novas estratégias sejam implantadas visando a transformação da tendência estacionária em decrescente.

\section{Referências}

Alagoas. Secretaria de Estado da Saúde de Alagoas. (2014). Relatórios da Análise de Situação de Saúde 2014. Saúde Alagoas -Análise da Situação de Saúde.

Alagoas. Secretaria de Estado da Saúde de Alagoas. (2017). Saúde Alagoas: Análise da Situação de Saúde 2017. Superintendência de Vigilância em Saúde. Gerência de Informação e Análise da Situação de Saúde.

Barbas, D. da S., Costa, A. J. L., Luiz, R. R., \& Kale, P. L. (2009). Determinantes do peso insuficiente e do baixo peso ao nascer na cidade do Rio de Janeiro, Brasil, 2001. Epidemiologia e Serviços de Saúde, 18(2), 161-170. https://doi.org/10.5123/S1679-49742009000200007

Benzies, K. M. (2008). Advanced maternal age: Are decisions about the timing of child-bearing a failure to understand the risks? CMAJ: Canadian Medical Association Journal = Journal de l'Association Medicale Canadienne, 178(2), 183-184. https://doi.org/10.1503/cmaj.071577

Blencowe, H., Krasevec, J., Onis, M. de, Black, R. E., An, X., Stevens, G. A., Borghi, E., Hayashi, C., Estevez, D., Cegolon, L., Shiekh, S., Hardy, V. P., Lawn, J. E., \& Cousens, S. (2019). National, regional, and worldwide estimates of low birthweight in 2015, with trends from 2000: A systematic analysis. The Lancet Global Health, 7(7), e849-e860. https://doi.org/10.1016/S2214-109X(18)30565-5 
Chermont, A., Miralha, A. L., Souza, L. E. C. de, \& Cunha, K. da C. (2019). Fatores associados ao baixo peso ao nascer em uma maternidade pública. Pará Research Medical Journal, 3(1), e03. https://doi.org/10.4322/prmj.2019.003

Cristovão, F., \& Pedraza, D. F. (2015). Tendência secular do peso ao nascer nas cidades de Cabedelo e Cacimbas, nordeste do Brasil. Espaço para Saúde, 16(1), 7-17. https://doi.org/10.22421/15177130-2015v16n1p7

de Souza Buriol, V. C., Hirakata, V., Goldani, M. Z., \& da Silva, C. H. (2016). Temporal evolution of the risk factors associated with low birth weight rates in Brazilian capitals (1996-2011). Population Health Metrics, 14, 15. https://doi.org/10.1186/s12963-016-0086-0

Franceschini, S. do C. C., Priore, S. E., Pequeno, N. P. F., Silva, D. G. da, \& Sigulem, D. M. (2003). Fatores de risco para o baixo peso ao nascer em gestantes de baixa renda. Revista de Nutrição, 16(2), 171-179. https://doi.org/10.1590/S1415-52732003000200004

Franciotti, D. L., Mayer, G. N., \& Cancelier, A. C. L. (2010). Fatores de risco para baixo peso ao nascer: Um estudo de caso-controle. Risk factors for low birth weight: A case-control study. 39(3), 7.

Guimarães, E. A. de A., \& Velásquez-Meléndez, G. (2002). Determinantes do baixo peso ao nascer a partir do Sistema de Informação sobre Nascidos Vivos em Itaúna, Minas Gerais. Revista Brasileira de Saúde Materno Infantil, 2(3), 283-290. https://doi.org/10.1590/S1519-38292002000300009

Kramer, M. S. (1987). Determinants of low birth weight: Methodological assessment and meta-analysis. Bulletin of the World Health Organization, 65(5), $663-737$.

Leguizamon Junior, T., Steffani, J. A., \& Bonamigo, E. L. (2013). Choice of route of childbirth: Expectation of pregnant women and obstetricians. Revista Bioética, 21(3), 509-517. https://doi.org/10.1590/S1983-80422013000300015

Minagawa, Á. T., Biagoline, R. E. M., Fujimori, E., Oliveira, I. M. V. de, Moreira, A. P. de C. A., \& Ortega, L. D. S. (2006). Baixo peso ao nascer e condições maternas no pré-natal. Revista da Escola de Enfermagem da USP, 40(4), 548-554. https://doi.org/10.1590/S0080-62342006000400014

Ministério da Saúde. (2021). DATASUS-Departamento de informática do SUS - Sistema de informações sobre nascidos vivos. http://www2.datasus.gov.br/DATASUS/index.php?area=0205\&id=6936\&VObj=http://tabnet.datasus.gov.br/cgi/deftohtm.exe?sinasc/cnv/nv

Moraes, A. B. de, Zanini, R. R., Riboldi, J., \& Giugliani, E. R. J. (2012). Risk factors for low birth weight in Rio Grande do Sul State, Brazil: Classical and multilevel analysis. Cadernos de Saúde Pública, 28(12), 2293-2305. https://doi.org/10.1590/S0102-311X2012001400008

Moreira, A. I. M., Sousa, P. R. M. de, Sarno, F., Moreira, A. I. M., Sousa, P. R. M. de, \& Sarno, F. (2018). Baixo peso ao nascer e seus fat ores associados. Einstein (São Paulo), 16(4). https://doi.org/10.31744/einstein_journal/2018ao4251

Nunes, M. de F. F. P. (2007). Fatores associados ao retardo de crescimento intra-uterino em recém nascidos em maternidades públicas da cidade de Salvador-Bahia. http://repositorio.ufba.br/ri/handle/ri/10403

Pedraza, D. F., Souza, M. M. de, Cristóvão, F. S., \& França, I. S. X. de. (2014). Baixo peso ao nascer no Brasil: Revisão sistemática de estudos baseados no sistema de informações sobre nascidos vivos. Pediatr. $\mathrm{mod}$, 50(2). bin/wxislind.exe/iah/online/?IsisScript=iah/iah.xis\&src=google\&base=LILACS\&lang=p\&nextAction=lnk\&exprSearch=718697\&indexSearch=ID

Pinto, J. R. (2010). Morbidade de crianças com baixo peso ao nascer durante o primeiro ano de vida na cidade de Sobral, Ceará.

PNUD, IPEA, \& FIP. (2020). Atlas Brasil. http://www.atlasbrasil.org.br/consulta/planilha

Santos, G. H. N. dos, Martins, M. da G., Sousa, M. da S., \& Batalha, S. de J. C. (2009). Impacto da idade materna sobre os resultados perinatais e via de parto. Revista Brasileira de Ginecologia e Obstetrícia, 31, 326-334. https://doi.org/10.1590/S0100-72032009000700002

Silva, M. A. P. da, Menezes, R. C. E. de, Oliveira, M. A. A., Longo-Silva, G., Asakura, L., Silva, M. A. P. da, Menezes, R. C. E. de, Oliveira, M. A. A., Longo-Silva, G., \& Asakura, L. (2014). Atenção Básica em Alagoas: Expansão da Estratégia Saúde da Família, do Nasf e do componente alimentação/nutrição. Saúde em Debate, 38(103), 720-732. https://doi.org/10.5935/0103-1104.20140066

Silva, T. R. S. R. da. (2003). O peso ao nascer no municipio de Presidente Prudente, São Paulo de 1998 a 2001 [Dissertação de mestrado, Universidade Federal de São Paulo (UNIFESP)]. https://repositorio.unifesp.br/handle/11600/18488

Viana, K. de J., Taddei, J. A. de A. C., Cocetti, M., \& Warkentin, S. (2013). Peso ao nascer de crianças brasileiras menores de dois anos. Cadernos de Saúde Pública, 29(2), 349-356. https://doi.org/10.1590/S0102-311X2013000200021

Weidle, W. G., Medeiros, C. R. G., Grave, M. T. Q., Dal Bosco, S. M., Weidle, W. G., Medeiros, C. R. G., Grave, M. T. Q., \& Dal Bosco, S. M. (2014). Choice of delivery method by the woman: Autonomy or induction? Cadernos Saúde Coletiva, 22(1), 46-53. https://doi.org/10.1590/1414462X2014000ALAGOAS. SECRETARIA DE ESTADO DA SAÚDE. (2014). Relatórios da Análise de Situação de Saúde 2014. Saúde Alagoas -Análise da Situação de Saúde.

Alagoas. Secretaria de Estado da Saúde de Alagoas. (2017). Saúde Alagoas: Análise da Situação de Saúde 2017. Superintendência de Vigilância em Saúde. Gerência de Informação e Análise da Situação de Saúde.

Barbas, D. da S., Costa, A. J. L., Luiz, R. R., \& Kale, P. L. (2009). Determinantes do peso insuficiente e do baixo peso ao nascer na cidade do Rio de Janeiro, Brasil, 2001. Epidemiologia e Serviços de Saúde, 18(2), 161-170. https://doi.org/10.5123/S1679-49742009000200007

Benzies, K. M. (2008). Advanced maternal age: Are decisions about the timing of child-bearing a failure to understand the risks? CMAJ: Canadian Medical Association Journal = Journal de l'Association Medicale Canadienne, 178(2), 183-184. https://doi.org/10.1503/cmaj.071577

Blencowe, H., Krasevec, J., Onis, M. de, Black, R. E., An, X., Stevens, G. A., Borghi, E., Hayashi, C., Estevez, D., Cegolon, L., Shiekh, S., Hardy, V. P., Lawn, J. E., \& Cousens, S. (2019). National, regional, and worldwide estimates of low birthweight in 2015, with trends from 2000: A systematic analysis. The Lancet Global Health, 7(7), e849-e860. https://doi.org/10.1016/S2214-109X(18)30565-5 
Chermont, A., Miralha, A. L., Souza, L. E. C. de, \& Cunha, K. da C. (2019). Fatores associados ao baixo peso ao nascer em uma maternidade pública. Pará Research Medical Journal, 3(1), e03. https://doi.org/10.4322/prmj.2019.003

Cristovão, F., \& Pedraza, D. F. (2015). Tendência Secular Do Peso Ao Nascer Nas Cidades De Cabedelo E Cacimbas, Nordeste Do Brasil. Espaço para Saúde, 16(1), 7-17. https://doi.org/10.22421/15177130-2015v16n1p7

de Souza Buriol, V. C., Hirakata, V., Goldani, M. Z., \& da Silva, C. H. (2016). Temporal evolution of the risk factors associated with low birth weight rates in Brazilian capitals (1996-2011). Population Health Metrics, 14, 15. https://doi.org/10.1186/s12963-016-0086-0

Franceschini, S. do C. C., Priore, S. E., Pequeno, N. P. F., Silva, D. G. da, \& Sigulem, D. M. (2003). Fatores de risco para o baixo peso ao nascer em gestantes de baixa renda. Revista de Nutrição, 16(2), 171-179. https://doi.org/10.1590/S1415-52732003000200004

Franciotti, D. L., Mayer, G. N., \& Cancelier, A. C. L. (2010). Fatores de risco para baixo peso ao nascer: Um estudo de caso-controle. Risk factors for low birth weight: A case-control study. 39(3), 7.

Guimarães, E. A. de A., \& Velásquez-Meléndez, G. (2002). Determinantes do baixo peso ao nascer a partir do Sistema de Informação sobre Nascidos Vivos em Itaúna, Minas Gerais. Revista Brasileira de Saúde Materno Infantil, 2(3), 283-290. https://doi.org/10.1590/S1519-38292002000300009

Kramer, M. S. (1987). Determinants of low birth weight: Methodological assessment and meta-analysis. Bulletin of the World Health Organization, 65(5), $663-737$

Leguizamon Junior, T., Steffani, J. A., \& Bonamigo, E. L. (2013). Choice of route of childbirth: Expectation of pregnant women and obstetricians. Revista Bioética, 21(3), 509-517. https://doi.org/10.1590/S1983-80422013000300015

Minagawa, Á. T., Biagoline, R. E. M., Fujimori, E., Oliveira, I. M. V. de, Moreira, A. P. de C. A., \& Ortega, L. D. S. (2006). Baixo peso ao nascer e condições maternas no pré-natal. Revista da Escola de Enfermagem da USP, 40(4), 548-554. https://doi.org/10.1590/S0080-62342006000400014

Ministério da Saúde. (2021). DATASUS-Departamento de informática do SUS - Sistema de informações sobre nascidos vivos. http://www2.datasus.gov.br/DATASUS/index.php?area=0205\&id=6936\&VObj=http://tabnet.datasus.gov.br/cgi/deftohtm.exe?sinasc/cnv/nv

Moraes, A. B. de, Zanini, R. R., Riboldi, J., \& Giugliani, E. R. J. (2012). Risk factors for low birth weight in Rio Grande do Sul State, Brazil: Classical and multilevel analysis. Cadernos de Saúde Pública, 28(12), 2293-2305. https://doi.org/10.1590/S0102-311X2012001400008

Moreira, A. I. M., Sousa, P. R. M. de, Sarno, F., Moreira, A. I. M., Sousa, P. R. M. de, \& Sarno, F. (2018). Baixo peso ao nascer e seus fatores associados. Einstein (São Paulo), 16(4). https://doi.org/10.31744/einstein_journal/2018ao4251

Nunes, M. de F. F. P. (2007). Fatores associados ao retardo de crescimento intra-uterino em recém nascidos em maternidades públicas da cidade de SalvadorBahia. http://repositorio.ufba.br/ri/handle/ri/10403

Pedraza, D. F., Souza, M. M. de, Cristóvão, F. S., \& França, I. S. X. de. (2014). Baixo peso ao nascer no Brasil: Revisão sistemática de estudos baseados no sistema de informações sobre nascidos vivos. Pediatr. bin/wxislind.exe/iah/online/?IsisScript=iah/iah.xis\&src=google\&base=LILACS\&lang=p\&nextAction=lnk\&exprSearch=718697\&indexSearch=ID

Pinto, J. R. (2010). Morbidade de crianças com baixo peso ao nascer durante o primeiro ano de vida na cidade de Sobral, Ceará.

PNUD, IPEA, \& FIP. (2020). Atlas Brasil. http://www.atlasbrasil.org.br/consulta/planilha

Santos, G. H. N. dos, Martins, M. da G., Sousa, M. da S., \& Batalha, S. de J. C. (2009). Impacto da idade materna sobre os resultados perinatais e via de parto. Revista Brasileira de Ginecologia e Obstetrícia, 31, 326-334. https://doi.org/10.1590/S0100-72032009000700002

Silva, M. A. P. da, Menezes, R. C. E. de, Oliveira, M. A. A., Longo-Silva, G., Asakura, L., Silva, M. A. P. da, Menezes, R. C. E. de, Oliveira, M. A. A., Longo-Silva, G., \& Asakura, L. (2014). Atenção Básica em Alagoas: Expansão da Estratégia Saúde da Família, do Nasf e do componente alimentação/nutrição. Saúde em Debate, 38(103), 720-732. https://doi.org/10.5935/0103-1104.20140066

Silva, T. R. S. R. da. (2003). O peso ao nascer no municipio de Presidente Prudente, São Paulo de 1998 a 2001 [Dissertação de mestrado, Universidade Federal de São Paulo (UNIFESP)]. https://repositorio.unifesp.br/handle/11600/18488

Viana, K. de J., Taddei, J. A. de A. C., Cocetti, M., \& Warkentin, S. (2013). Peso ao nascer de crianças brasileiras menores de dois anos. Cadernos de Saúde Pública, 29(2), 349-356. https://doi.org/10.1590/S0102-311X2013000200021

Weidle, W. G., Medeiros, C. R. G., Grave, M. T. Q., Dal Bosco, S. M., Weidle, W. G., Medeiros, C. R. G., Grave, M. T. Q., \& Dal Bosco, S. M. (2014). Choice of delivery method by the woman: Autonomy or induction? Cadernos Saúde Coletiva, 22(1), 46-53. https://doi.org/10.1590/1414-462X201400010008

WHO- World Health Organization. (2014). World health statistics 2014. World Health Organization. https://apps.who.int/iris/handle/10665/112738

World Health Organization - WHO. (1995). WHO | Physical status: The use and interpretation of anthropometry. WHO; World Health Organization. https://www.who.int/childgrowth/publications/physical_status/en/

Zambonato, A. M. K., Pinheiro, R. T., Horta, B. L., \& Tomasi, E. (2004). Fatores de risco para nascimento de crianças pequenas para idade gestacional. Revista de Saúde Pública, 38(1), 24-29. https://doi.org/10.1590/S0034-8910200400010000410008

World Health Organization- WHO. (2014). World health statistics 2014. World Health Organization. https://apps.who.int/iris/handle/10665/112738

World Health Organization - WHO. (1995). WHO | Physical status: The use and interpretation of anthropometry. WHO; World Health Organization. https://www.who.int/childgrowth/publications/physical_status/en/

Zambonato, A. M. K., Pinheiro, R. T., Horta, B. L., \& Tomasi, E. (2004). Fatores de risco para nascimento de crianças pequenas para idade gestacional. Revista de Saúde Pública, 38(1), 24-29. https://doi.org/10.1590/S0034-89102004000100004 